\title{
Use of microbiological and patient data for choice of empirical antibiotic therapy in acute cholangitis
}

Tassilo Kruis ${ }^{1,2 *}$, Sarah Güse-Jaschuck², Britta Siegmund², Thomas Adam ${ }^{1}$ and Hans-Jörg Epple²

\begin{abstract}
Background: Ineffective antibiotic therapy increases mortality of acute cholangitis. The choice of antibiotics should reflect local resistance patterns and avoid the overuse of broad-spectrum agents. In this study, we analysed how results of bile and blood cultures and patient data can be used for selection of empirical antibiotic therapy in acute cholangits.
\end{abstract}

Methods: Pathogen frequencies and susceptibility rates were determined in 423 positive bile duct cultures and 197 corresponding blood cultures obtained from 348 consecutive patients with acute cholangitis. Patient data were retrieved from the medical records. Associations of patient and microbiological data were assessed using the Chi-2 test and multivariate binary logistic regression.

Results: In bile cultures, enterobacterales and enterococci were isolated with equal frequencies of approximately $30 \%$ whereas in blood cultures, enterobacterales predominated (56\% compared to $21 \%$ enterococci). Antibiotic resistance rates of enterobacterales were $>20 \%$ for fluorochinolones, cephalosporines and acylureidopenicillins but not for carbapenems (<2\%). The efficacy of empirical therapy was poor with a coverage of bacterial bile and blood culture isolates in 51 and 69\%, respectively. By multivariate analysis, predictors for pathogen species, antibiotic susceptibility and expected antibiotic coverage were identified.

Conclusions: In unselected patients treated for acute cholangitis in a large tertiary refferential center, use of carbapenems seems necessary to achieve a high antibiotic coverage. However, by analysis of patient and microbiological data, subgroups for highly effective carbapenem-sparing therapy can be defined. For patients with community-acquired cholangitis without biliary prosthesis who do not need intensive care, piperacillin/tazobactam represents a regimen with an expected excellent antibiotic coverage.

Keywords: Acute cholangitis, Biliary tract infection, Gastrointestinal tract microbiology, Antimicrobial resistance, Empirical antibiotic therapy, Carbapenem-sparing therapy

\footnotetext{
* Correspondence: tassilo.kruis@laborberlin.com

'Labor Berlin Charité Vivantes GmbH, Mikrobiologie \& Hygiene, Berlin, Germany

${ }^{2}$ Charité - Universitätsmedizin Berlin, Medizinische Klinik für Gastroenterologie, Infektiologie und Rheumatologie, Campus Benjamin Franklin, Berlin, Germany
}

(c) The Author(s). 2020 Open Access This article is licensed under a Creative Commons Attribution 4.0 International License, which permits use, sharing, adaptation, distribution and reproduction in any medium or format, as long as you give appropriate credit to the original author(s) and the source, provide a link to the Creative Commons licence, and indicate if changes were made. The images or other third party material in this article are included in the article's Creative Commons licence, unless indicated otherwise in a credit line to the material. If material is not included in the article's Creative Commons licence and your intended use is not permitted by statutory regulation or exceeds the permitted use, you will need to obtain permission directly from the copyright holder. To view a copy of this licence, visit http://creativecommons.org/licenses/by/4.0/ The Creative Commons Public Domain Dedication waiver (http://creativecommons.org/publicdomain/zero/1.0/) applies to the data made available in this article, unless otherwise stated in a credit line to the data. 


\section{Background}

Acute cholangitis is a potentially life-threatening infection of the biliary tract. The main underlying pathological process is obstruction of bile ducts leading to cholestasis and impeded clearance of intestinal microorganisms ascending from the duodenum. The increase in biliary tract pressure and the local inflammatory response to the infecting organisms impair the integrity of the biliary epithelium, facilitate bacterial translocation into the circulation, and promote systemic inflammation [1]. In addition, certain procedures, such as sphincterotomy, stent placement or biliodigestive anastomosis, can predispose to bacteriobilia and cholangitis. In these situations, infection of the biliary tract may develop in the absence of obstruction $[1,2]$.

The clinical course of acute cholangitis ranges from mild abdominal symptoms to sepsis and shock. Despite considerable progress in the treatment of acute cholangitis during the last decades, recent studies still report mortality rates of up to $10 \%[2,3]$. As with other localized infections, treatment success depends on efficient source control and appropriate antibiotic therapy. Source control is achieved by biliary decompression by endoscopic or percutaneous drainage. The importance of antibiotic therapy is highlighted by the fact that inappropriate empirical therapy is associated with increased mortality [3].

Studies from different geographic regions of the world consistently found E.coli, Klebsiella spp. and Enterococcus spp. as most common pathogens in ascending cholangitis [4]. Thus, the microbial spectrum to be covered by empirical therapy seems to be well defined in general. However, individual patient factors as well as local resistance patterns can increase the probability for infection with resistant enterobacterales, P.aeruginosa or other difficult-to-treat pathogens [5]. Enterobacterales resistant to third generation cephalosporines (3GCRE) are of particular importance because their treatment regularly requires the use of carbapenems. Because of the rising prevalence of cephalosporine resistance, the clinician is confronted with the dilemma of ensuring broad empirical antibiotic coverage while at the same time avoiding excessive use of carbapenems. Particularly in institutions with a high prevalence of 3GCRE, it seems difficult to define a role for carbapenem-sparing regimens. Knowledge of local susceptibility rates alone is not sufficient to solve this problem.

In our study, we investigated whether the analysis of patient factors and their association with pathogens and antibiotic resistance could be helpful to avoid overuse of carbapenems and other broad-spectrum antibiotics. To this end, we conducted a retrospective analysis of 348 patients treated for ascending cholangitis in Germany's largest university medical center. We determined the pathogen spectrum and antibiotic susceptibilities of enterobacterales and enterococci in biliary and blood samples from these patients. Particularly, we analysed the significance of various patient factors for predicting the isolation of bacterial biliary pathogens and their antibiotic susceptibility profile. Our results demonstrate, how the combination of microbiological and patient data can be used for the choice of efficacious antibiotic therapy of patients with acute cholangitis.

\section{Methods}

All consecutive specimens sent to the microbiological laboratory of the Charite (Institute of Microbiology and Hygiene of the Charité until December 2010; Labor Berlin Charité Vivantes $\mathrm{GmbH}$, thereafter) from January 2007 until July 2015 were screened for positive bile cultures obtained by endoscopic or percutaneous sampling. From all patients with positive bile cultures, blood culture results were also extracted, if they had been collected within $48 \mathrm{~h}$ from the corresponding bile sample.

The diagnosis of cholangitis was reviewed against the patient records. Bile cultures that were not collected within a course of cholangitis were excluded. Samples were also excluded, if the patient's age was $<18$ years or clinical data were incomplete or inconsistent. Figure 1 depicts the schematic structure of the study.

Bile specimens were cultivated on conventional solid media and incubated in aerobic and anaerobic atmospheres at $37^{\circ} \mathrm{C}$. Aerobic microorganisms were routinely identified and tested for antibiotic susceptibility using the VITEK 2 System (bioMérieux, France). Antibiotic susceptibilities were interpreted according to EUCAST recommendations (http://www.eucast.org/). If necessary, additional commercial methods were applied such as matrix-associated laser desorption/ionization time-offlight mass spectrometry (MALDI-MS; Bruker, Massachusetts, USA, before 2011, or bioMérieux, France, since 2011) for species identification or the disk diffusion method and E-tests for antibiotic susceptibility testing. Anaerobes were identified by MALDI-MS and tested for susceptibility using ATB ANA (bioMérieux, France) or Etests. For this study, "intermediate" (I) results in antimicrobial sensitivity testing (AST) were considered "resistant" (R). For enterobacterales, AST was done using ampicillin/sulbactam, piperacillin/tazobactam, cefotaxime, meropenem, and ciprofloxacin. Enterococcus strains were investigated on ampicillin and vancomycin resistance, respectively. Blood cultures with Coagulase-negative Staphylococci (CoNS) in only one pair of bottles were considered contaminated. In line with the recommendations of the German Commission for Hospital Hygiene and Infection Prevention at the Robert Koch Institute (KRINKO) [6] enterobacterales, $P$. aeruginosa, and $A$. baumannii complex isolates were classified as multi-resistant 


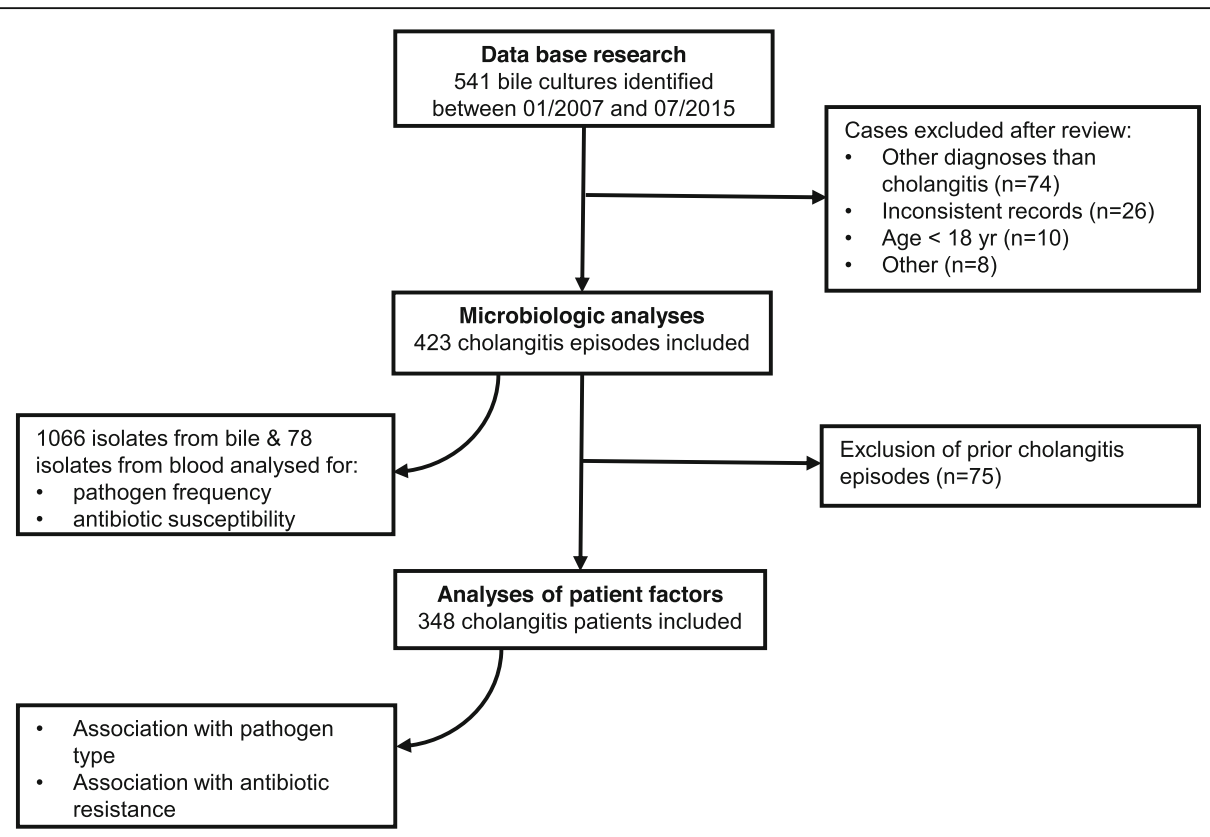

Fig. 1 Study design

gram-negative rods (MRGN) if they were resistant to three or more of the following substance classes: acylureidopenicillins, third generation cephalosporins, carbapenems, fluorochinolones.

Patient data were extracted from the hospital records. In patients hospitalized several times for recurrent cholangitis, only the most recent episode was analysed. Also, in patients with more than one procedure of bile sampling during the same hospital stay, only culture results obtained during the first sampling procedure were considered for correlation of patient factors with microbiological data. Data on empirical antibiotic treatment were available for 229 patients.

The statistical evaluation was carried out after consultation with the Institute for Biometry and Clinical Epidemiology of the Charité - Universitätsmedizin Berlin using SPSS version 23.0. Significant differences in categorical variables were assessed using the Chi-2 test. Predictors with $p \leq 0.2$ in the univariate analysis were then tested using multivariate binary logistic regression through backward elimination and forward selection. The significance level in two-sided testing was $p<0.05$.

\section{Ethics, consent and permissions}

The study was approved by the internal review board (local ethics committee of Charité - Universitätmedizin Berlin; registry number EA2/033/16). The need for informed consent was waived for this retrospective study.

\section{Results}

Microbiological spectrum and antibiotic susceptibilities

Overall, 541 bile cultures sampled within the study period were identified. After checking for inclusion and exclusion criteria, 118 cases were excluded (Fig. 1). From the remaining 423 bile duct cultures, a total of $1066 \mathrm{mi}$ croorganisms (bacteria or fungi) were isolated with polymicrobial growth detected in $69 \%$ of cultures. The most

Table 1 Frequency of microorganisms isolated in bile and blood

\begin{tabular}{lll}
\hline & $\begin{array}{l}\text { Bile } \\
(n=1066)\end{array}$ & $\begin{array}{l}\text { Blood } \\
(n=78)\end{array}$ \\
\hline Enteroccus spp. & $31.0 \%$ & $20.5 \%$ \\
Enterobacterales & $29.5 \%$ & $56.4 \%$ \\
E.coli & $13.2 \%$ & $32.1 \%$ \\
Klebsiella spp. & $8.0 \%$ & $14.1 \%$ \\
Enterobacter spp. & $2.9 \%$ & $5.1 \%$ \\
Citrobacter spp. & $2.4 \%$ & $2.6 \%$ \\
Other enterobacterales & $3.0 \%$ & $2.6 \%$ \\
Candida spp. & $16.9 \%$ & $3.8 \%$ \\
Streptococcus spp. & $6.6 \%$ & $1.3 \%$ \\
Anaerobes & $4.9 \%$ & $1.3 \%$ \\
CoNS & $3.3 \%$ & $3.8 \%$ \\
P. aeruginosa & $2.3 \%$ & $7.7 \%$ \\
Other & $5.5 \%$ & $6.4 \%$ \\
\hline
\end{tabular}

$\mathrm{n}$, number of isolates from bile and blood, respectively CoNS, Coagulase-negative staphylococci 
frequent pathogens isolated were enterococci (31\%) and enterobacterales (30\%), followed by Candida spp. (17\%), streptococci (7\%), and anaerobes (5\%). P. aeruginosa represented $2 \%$ of bile culture isolates (Table 1 ). For the purpose of this study, only bacterial pathogens were considered for further evaluation.

We identified 197 blood cultures collected within $48 \mathrm{~h}$ of the respective bile samples. Seventy of these had turned positive. After exclusion of CoNS contaminants (8/70), the rate of positive blood cultures was $32 \%$ (62/197). Polymicrobial growth was less frequent in blood than in bile cultures (16\% versus 69\%, respectively). Complete or partial pathogen concordance with corresponding bile cultures was present in $65 \%$ (40/62). While in bile cultures entorobacterales and enterococci were isolated with similar frequencies (approximately 30\%), enterobacterales comprised the majority of isolates in blood cultures (56\% compared to $21 \%$ enterococci). In addition, P.aeruginosa was isolated more frequently in blood cultures compared to bile cultures ( $8 \%$ vs. $2 \%$ ) (Table 1 ). With the exception of carbapenems, a high level of resistance against all classes of antibiotics tested was found in enterobacterales isolated from bile or blood. Also, at least one third of enterococci isolated from bile and blood were resistant to ampicillin (Table 2).

\section{Patient factors}

Considering only the most recent and excluding previous cholangitis episodes, 348 patients with acute cholangitis were included into the analysis of patient factors. As shown in Table 3, patients were characterized by a high burden of comorbidity, a high prevalence of preexisting biliary tract pathologies and a high prevalence of prior biliary tract interventions, reflecting the patient selection expected for a tertiary reference center with associated liver transplant unit. Almost $60 \%$ of patients included had an indwelling biliary drainage, almost 30\%

Table 2 Antibiotic resistance of bile and blood culture isolates

\begin{tabular}{|c|c|c|c|c|}
\hline & \multicolumn{2}{|l|}{ Bile } & \multicolumn{2}{|l|}{ Blood } \\
\hline & & $(n / N)$ & & $(n / N)$ \\
\hline \multicolumn{5}{|l|}{ Enterobacterales } \\
\hline Ampicillin/sulbactam & $54.2 \%$ & $167 / 308$ & $56.8 \%$ & $25 / 44$ \\
\hline Piperacillin/tazobactam & $34.0 \%$ & $103 / 303$ & $25.0 \%$ & $11 / 44$ \\
\hline Cefotaxime & $26.9 \%$ & $83 / 308$ & $11.4 \%$ & $5 / 44$ \\
\hline Meropenem & $1.9 \%$ & $6 / 310$ & $2.3 \%$ & $1 / 44$ \\
\hline Ciprofloxacin & $20.0 \%$ & $62 / 310$ & $29.5 \%$ & $13 / 44$ \\
\hline \multicolumn{5}{|l|}{ Enterococcus spp. } \\
\hline Ampicillin & $33.1 \%$ & $103 / 311$ & $37.5 \%$ & $6 / 16$ \\
\hline Vancomycin & $13.2 \%$ & $40 / 302$ & $18.8 \%$ & $3 / 16$ \\
\hline
\end{tabular}

$\mathrm{n} / \mathrm{N}$, resistant isolates/tested isolates had a biliodigestive anastomosis, and more than $10 \%$ of patients have had liver transplantation (Table 3).

Overall, enterobacterales were detected in the bile of $52 \%(182 / 348)$ of patients. In the univariate analysis, enterobacterales were isolated less frequently, if bile sampling was performed later than $48 \mathrm{~h}$ after admission, after percutaneous compared to endoscopic sampling, in patients with previous or ongoing ICU treatment (during the current hospital stay), and in patients with biliodigestive anastomosis or transplanted liver (Table 4). Enterococci were detected in 59\% (205/348) of patients. They were detected more frequently in patients with a high level of comorbidity (as indicated by a Charlson index $\geq 5$ ), antibiotic pretreatment, prior biliary tract intervention, indwelling biliary drainage, and biliary stenosis. P. aeruginosa was detected in 6\% (21/348) of patients. The only risk factor associated with isolation of $P$. aeruginosa in the univariate analysis was ICU treatment.

After multivariate analysis, bile sampling later than 48 $\mathrm{h}$ after admission and ICU treatment before or whilst sampling remained negative predictors for isolation of enterobacterales from bile culture (OR 0.41 and 0.38 , respectively), whereas the presence of a biliary drainage remained a strong risk factor for the isolation of enterococci (OR 3.1) (Table 4). In line with this, a significant linear trend was observed between the number of interventions and the frequency of enterococcal infections ( $p \leq 0.001)$ amongst 260 patients in whom the exact numbers of previous biliary tract interventions could be determined (Fig. 2).

As to the association of patient factors with antibiotic resistance, multivariate analysis showed that male sex and MDR carrier status (comprising MRSA, MRGN, or VRE) were independently associated with an increased prevalence of both 3GCRE and MRGN in bile cultures (Table 4). In $41 \%$ of individuals with MRGN carrier status, MRGN were also cultured from bile sampled during acute cholangitis (compared to only $8 \%$ in individuals without, $\mathrm{p} \leq 0.001$ ). In addition, a high level of comorbidity was associated with an increase in 3GCRE, while percutaneous bile sampling and the presence of a biliary stenosis was associated with more MRGN (Table 4). By linear trend, patients with more than three biliary tract interventions were significantly more likely to present with 3GCRE or MRGN biliary tract infections (Fig. 2). Regarding VRE, 20\% of known carriers also had VRE isolated from bile cultures during acute cholangitis (compared to $7 \%$ of patients without carrier status).

\section{Antibiotic therapy}

In 229 patients the empirical antibiotic regimen could be determined from the clinical records. One hundred thirty-six patients (59\%) received monotherapy, and 93 
Table 3 Patient characterisitics

\begin{tabular}{|c|c|c|}
\hline \multirow[t]{2}{*}{ Total study population } & \multicolumn{2}{|l|}{$N=348$} \\
\hline & & (n) \\
\hline \multirow[t]{2}{*}{ Male } & $63.8 \%$ & 222 \\
\hline & median & $\min . / \max$ \\
\hline Age at intervention (years) & 64 & $18 / 94$ \\
\hline Age-adjusted Charlson index & 5 & $0 / 12$ \\
\hline Cardiovascular disease & $42.2 \%$ & 147 \\
\hline Malignoma & $40.5 \%$ & 141 \\
\hline Liver disease & $27.6 \%$ & 96 \\
\hline Diabetes & $24.1 \%$ & 84 \\
\hline Kidney disease & $20.4 \%$ & 71 \\
\hline Pulmonary disease & $15.2 \%$ & 53 \\
\hline Neurologic disorder & $8.6 \%$ & 30 \\
\hline MDR colonization & $15.6 \%$ & 59 \\
\hline Preexisting biliary tract pathologies ${ }^{a}$ & $83.0 \%$ & 289 \\
\hline Papillotomy & $30.7 \%$ & 107 \\
\hline Malignant stenosis & $30.5 \%$ & 106 \\
\hline Biliodigestive anastomosis & $28.7 \%$ & 100 \\
\hline Choledocholithiasis & $23.9 \%$ & 83 \\
\hline Benign stenosis & $23.6 \%$ & 82 \\
\hline Liver transplatation & $13.2 \%$ & 46 \\
\hline \multicolumn{3}{|l|}{ Prior biliary tract interventions } \\
\hline Yes & $81.0 \%$ & 282 \\
\hline No & $18.4 \%$ & 64 \\
\hline \multirow[t]{2}{*}{ N.d. } & $0.6 \%$ & 2 \\
\hline & median & $\min . / \max$ \\
\hline Number of prior interventions ${ }^{\mathrm{b}}$ & 2 & $0 / 32$ \\
\hline \multicolumn{3}{|l|}{ Preexisting biliary tract drainage } \\
\hline Yes & $58.9 \%$ & 205 \\
\hline No & $41.1 \%$ & 143 \\
\hline N.d. & $8.0 \%$ & 28 \\
\hline \multicolumn{3}{|l|}{ Antibiotic pre-treatment within $90 \mathrm{~d}$} \\
\hline Yes & $46.6 \%$ & 162 \\
\hline No & $45.4 \%$ & 158 \\
\hline N.d. & $8.0 \%$ & 28 \\
\hline \multicolumn{3}{|l|}{ Indication for recent intervention } \\
\hline Stenosis & $25.9 \%$ & 90 \\
\hline Choledocholithiasis & $21.0 \%$ & 73 \\
\hline Catheter related & $14.4 \%$ & 50 \\
\hline Drainage after LTX & $8.0 \%$ & 28 \\
\hline Liver abscess & $4.9 \%$ & 17 \\
\hline Pancreatitis & $4.6 \%$ & 16 \\
\hline Other & $21.3 \%$ & 74 \\
\hline \multicolumn{3}{|l|}{ Route of biliary drainage } \\
\hline Endoscopic & $58.3 \%$ & 203 \\
\hline
\end{tabular}

Table 3 Patient characterisitics (Continued)

\begin{tabular}{lll}
\hline Total study population & \multicolumn{1}{l}{$\mathbf{N} \mathbf{3 4 8}$} \\
\cline { 2 - 3 } & & (n) \\
\hline Percutaneous & $40.5 \%$ & 141 \\
N.d. & $1.1 \%$ & 4 \\
Successful biliary decompression & & \\
$\quad$ Yes & $92.8 \%$ & 323 \\
No & $3.4 \%$ & 12 \\
N.d. & $3.7 \%$ & 13 \\
Outcome & & \\
Death & $14.4 \%$ & 50 \\
Admission to ICU & $37.6 \%$ & 131 \\
& median & \\
Length of hospital stay (days) & 16.5 & $1 / 367$ \\
\hline
\end{tabular}

N.d., not determinable/not documented

MDR, multi-drug resistant bacteria including MRSA, MRGN, and VRE

${ }^{a}$ One or more underlying conditions possible in one individual

${ }^{b}$ The exact number of prior biliary tract interventions was known in 260 cases

patients (41\%) were treated with a combinational regimen. The most commonly prescribed substances were cephalosporines, followed by piperacillin/tazobactam and fluorochinolones (Table 5). The most frequent combinational therapy consisted of a beta-lactam plus metronidazole. With the regimens employed, bacterial organisms isolated from bile were covered in only 51\% of patients (taking into account all isolates of a sample). Even more importantly, empiric coverage of blood culture isolates was only 69\%. Amongst patients with bacteremia, three out of 11 (27\%) patients with inadequate empirical therapy deceased, compared to only two out of $22(8 \%)$ patients with adequate therapy.

The primary goals of antibiotic therapy in ascending cholangitis are to prevent and treat systemic spread of the infection. In order to test whether carbapenem-sparing antibiotic therapy might be used at least for certain selected patients, we analysed antibiotic coverage in patient subgroups defined by simple patient-associated factors. For this analysis, the efficacy of the antibiotic therapy was evaluated in respect to the blood culture isolates, because a positive blood culture obviously represents proof of systemic spread. As shown in Table 6, blood culture isolates obtained from patients with community-acquired cholangitis (biliary sampling within $48 \mathrm{~h}$ of admission), without indwelling biliary drainage, and no ICU treatment would have been covered by piperacillin/tazobactam by $100 \%$. In comparison, only a $78 \%$ coverage rate would have been achieved by third generation cephalosporines. In these patients, the use of carbapenems does not further improve antibiotic coverage.

\section{Discussion}

Empirical therapy of cholangitis represents an educated guess based on spectrum and likelihood of causative 
Table 4 Association between patient factors and pathogen frequencies

\begin{tabular}{|c|c|c|c|c|c|c|c|c|c|c|c|c|}
\hline \multicolumn{13}{|l|}{ Univariate analysis } \\
\hline & & \multirow[b]{2}{*}{$\mathrm{n}$} & \multicolumn{2}{|c|}{ Enterococcus spp. } & \multicolumn{2}{|c|}{ Enterobacterales } & \multicolumn{2}{|l|}{ 3GCRE } & \multicolumn{2}{|l|}{ MRGN } & \multicolumn{2}{|c|}{ P. aeruginosa } \\
\hline & & & & $p$-value & & $p$-value & & $p$-value & & $p$-value & & $p$-value \\
\hline \multirow[t]{2}{*}{ Age } & $<65 y$ & 180 & $57.8 \%$ & 0.664 & $48.3 \%$ & 0.134 & $11.2 \%$ & 0.621 & $9.0 \%$ & 0.592 & $5.6 \%$ & 0.823 \\
\hline & $>=65 y$ & 168 & $60.1 \%$ & & $56.5 \%$ & & $13.5 \%$ & & $10.8 \%$ & & $6.5 \%$ & \\
\hline \multirow[t]{2}{*}{ Sex } & $m$ & 222 & $58.6 \%$ & 0.910 & $53.2 \%$ & 0.738 & $15.6 \%$ & 0.016 & $13.3 \%$ & 0.008 & $7.2 \%$ & 0.252 \\
\hline & $f$ & 126 & $59.5 \%$ & & $50.8 \%$ & & $6.5 \%$ & & $4.0 \%$ & & $4.0 \%$ & \\
\hline \multirow[t]{2}{*}{ Charlson Index } & $<5$ & 201 & $53.7 \%$ & 0.027 & $50.2 \%$ & 0.384 & $8.6 \%$ & 0.019 & $7.5 \%$ & 0.100 & $5.0 \%$ & 0.366 \\
\hline & $>=5$ & 146 & $65.8 \%$ & & $55.5 \%$ & & $17.4 \%$ & & $13.2 \%$ & & $7.5 \%$ & \\
\hline \multirow[t]{2}{*}{ MDR carrier } & no & 289 & $57.8 \%$ & 0.386 & $51.6 \%$ & 0.570 & $9.1 \%$ & $\leq 0.001$ & $7.3 \%$ & $\leq 0.001$ & $5.2 \%$ & 0.224 \\
\hline & yes & 59 & $64.4 \%$ & & $55.9 \%$ & & $28.1 \%$ & & $22.8 \%$ & & $10.2 \%$ & \\
\hline \multirow[t]{2}{*}{ Time of bile collection from admission } & $\leq 48 h$ & 153 & $59.5 \%$ & 1.000 & $69.3 \%$ & $\leq 0.001$ & $13.9 \%$ & 0.509 & $7.2 \%$ & 0.147 & $4.6 \%$ & 0.488 \\
\hline & $>48 h$ & 190 & $58.9 \%$ & & $38.9 \%$ & & $11.3 \%$ & & $12.3 \%$ & & $6.8 \%$ & \\
\hline \multirow[t]{2}{*}{ Route of bile collection } & endoscopic & 203 & $56.7 \%$ & 0.438 & $60.1 \%$ & $\leq 0,001$ & $11.0 \%$ & 0.402 & $6.0 \%$ & 0.003 & $5.4 \%$ & 0.648 \\
\hline & percutaneous & 141 & $61.0 \%$ & & $42.6 \%$ & & $14.5 \%$ & & $15.8 \%$ & & $7.1 \%$ & \\
\hline \multirow[t]{2}{*}{$\mathrm{ICU}^{\mathrm{a}}$} & no & 226 & $59.7 \%$ & 0.732 & $63.3 \%$ & $\leq 0.001$ & $14.0 \%$ & 0.228 & $8.9 \%$ & 0.451 & $3.1 \%$ & 0.002 \\
\hline & yes & 122 & $57.4 \%$ & & $32.0 \%$ & & $9.1 \%$ & & $11.7 \%$ & & $11.5 \%$ & \\
\hline \multirow[t]{2}{*}{ Antibiotic pre-treatment ${ }^{b}$} & no & 157 & $52.5 \%$ & 0.006 & $56.3 \%$ & 0.372 & $13.0 \%$ & 1.000 & $7.0 \%$ & 0.179 & $4.4 \%$ & 0.345 \\
\hline & yes & 160 & $67.9 \%$ & & $51.2 \%$ & & $12.4 \%$ & & $11.9 \%$ & & $7.4 \%$ & \\
\hline \multirow[t]{2}{*}{ Prior biliary tract intervention } & no & 64 & $42.2 \%$ & 0.003 & $46.9 \%$ & 0.406 & $4.8 \%$ & 0.054 & $3.2 \%$ & 0.059 & $4.7 \%$ & 0.777 \\
\hline & yes & 282 & $63.1 \%$ & & $53.5 \%$ & & $14.1 \%$ & & $11.5 \%$ & & $6.4 \%$ & \\
\hline \multirow[t]{2}{*}{ Indwelling biliary tract drainage } & no & 143 & $43.4 \%$ & $\leq 0.001$ & $51.7 \%$ & 0.913 & $7.8 \%$ & 0.044 & $7.1 \%$ & 0.198 & $5.6 \%$ & 0.823 \\
\hline & yes & 205 & $69.8 \%$ & & $52.7 \%$ & & $15.4 \%$ & & $11.8 \%$ & & $6.3 \%$ & \\
\hline \multirow[t]{2}{*}{ Stenosis } & no & 161 & $50.9 \%$ & 0.006 & $47.8 \%$ & 0.133 & $8.2 \%$ & 0.033 & $5.7 \%$ & 0.018 & $5.0 \%$ & 0.503 \\
\hline & yes & 187 & $65.8 \%$ & & $56.1 \%$ & & $15.8 \%$ & & $13.4 \%$ & & $7.0 \%$ & \\
\hline \multirow[t]{2}{*}{ Biliodigestive anastomosis } & no & 248 & $59.5 \%$ & 0.803 & $57.5 \%$ & $\leq 0.001$ & $11.5 \%$ & 0.586 & $7.7 \%$ & 0.044 & $5.6 \%$ & 0.804 \\
\hline & yes & 100 & $57.3 \%$ & & $37.1 \%$ & & $14.1 \%$ & & $15.3 \%$ & & $7.0 \%$ & \\
\hline \multirow[t]{2}{*}{ Liver transplantation } & no & 302 & $60.3 \%$ & 0.201 & $56.6 \%$ & $\leq 0.001$ & $12.8 \%$ & 0.628 & $9.7 \%$ & 0.788 & $6.6 \%$ & 0.333 \\
\hline & yes & 46 & $50.0 \%$ & & $23.9 \%$ & & $8.7 \%$ & & $11.1 \%$ & & $2.2 \%$ & \\
\hline
\end{tabular}

Multivariate analysis - independent predictors

\section{OR $(95 \% \mathrm{Cl})$}

$3.11(1.94-5.00)$

$0.41(0.25-0.66)$

$0.38(0.23-0.63)$

$2.61(1.15-5.95)$

$1.98(1.00-3.92)$

$3.57(1.73-7.37)$

$4.27(1.42-12.82)$

$2.47(1.04-5.86)$

$2.61(1.13-6.01)$

$2.66(1,02-6,95)$

$4.06(1.59-10.34)$ $p$-value

$\leq 0.001$

$\leq 0.001$

$\leq 0.001$

0.022

0.049

$\leq 0.001$

0.010

0.040

0.024

0.046

Stenosis

P. aeruginosa

MRGN, multidrug resistant gram negative bacteria as defined by KRINKO [6]

MDR, multi-drug resistant bacteria including MRSA, MRGN, and VRE

a ICU, defined as treatment on ICU before or during bile sampling

${ }^{\mathrm{b}}$ Antibiotic pre-treatment within 3 months prior to admisson 


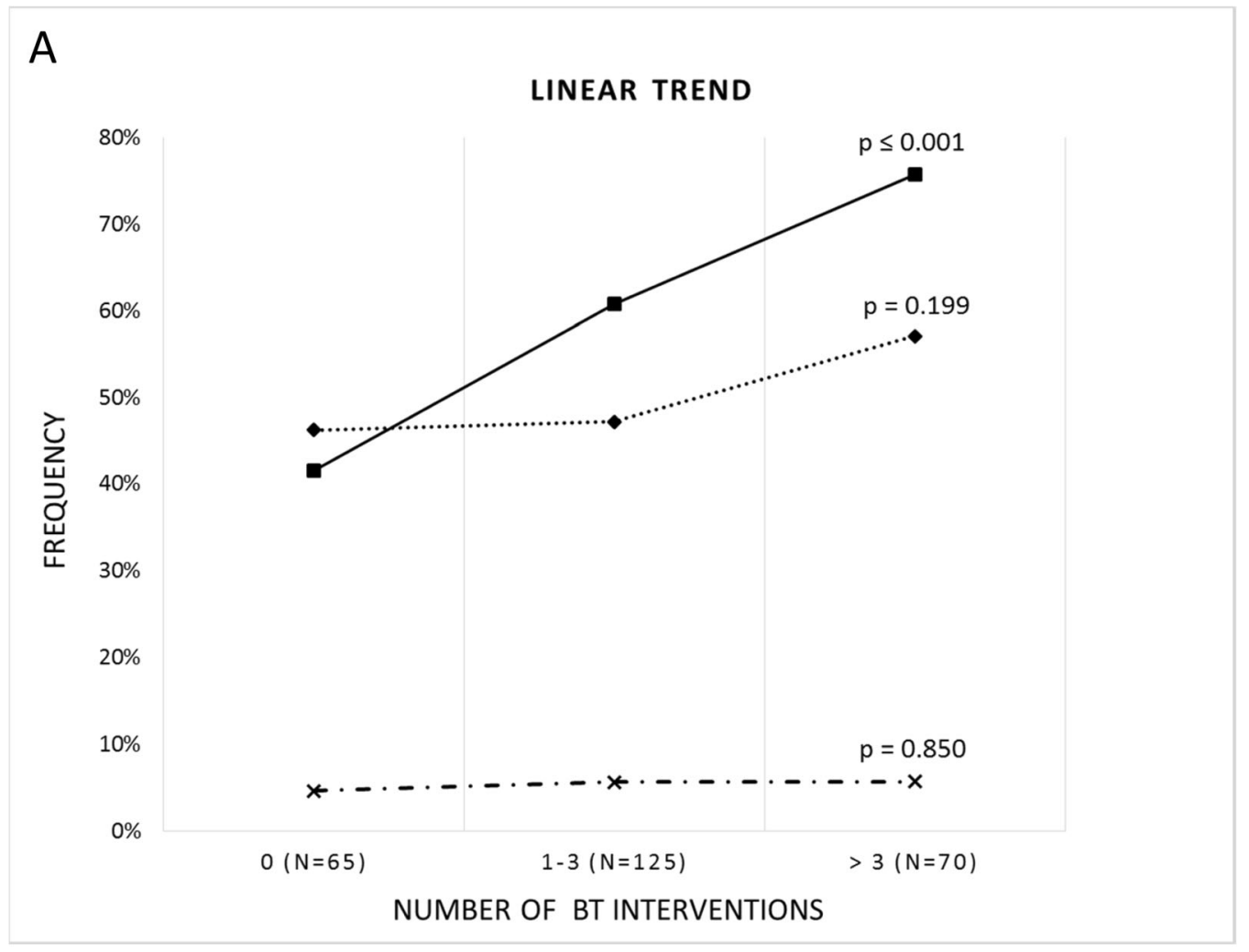

B

LINEAR TREND

$25 \%$

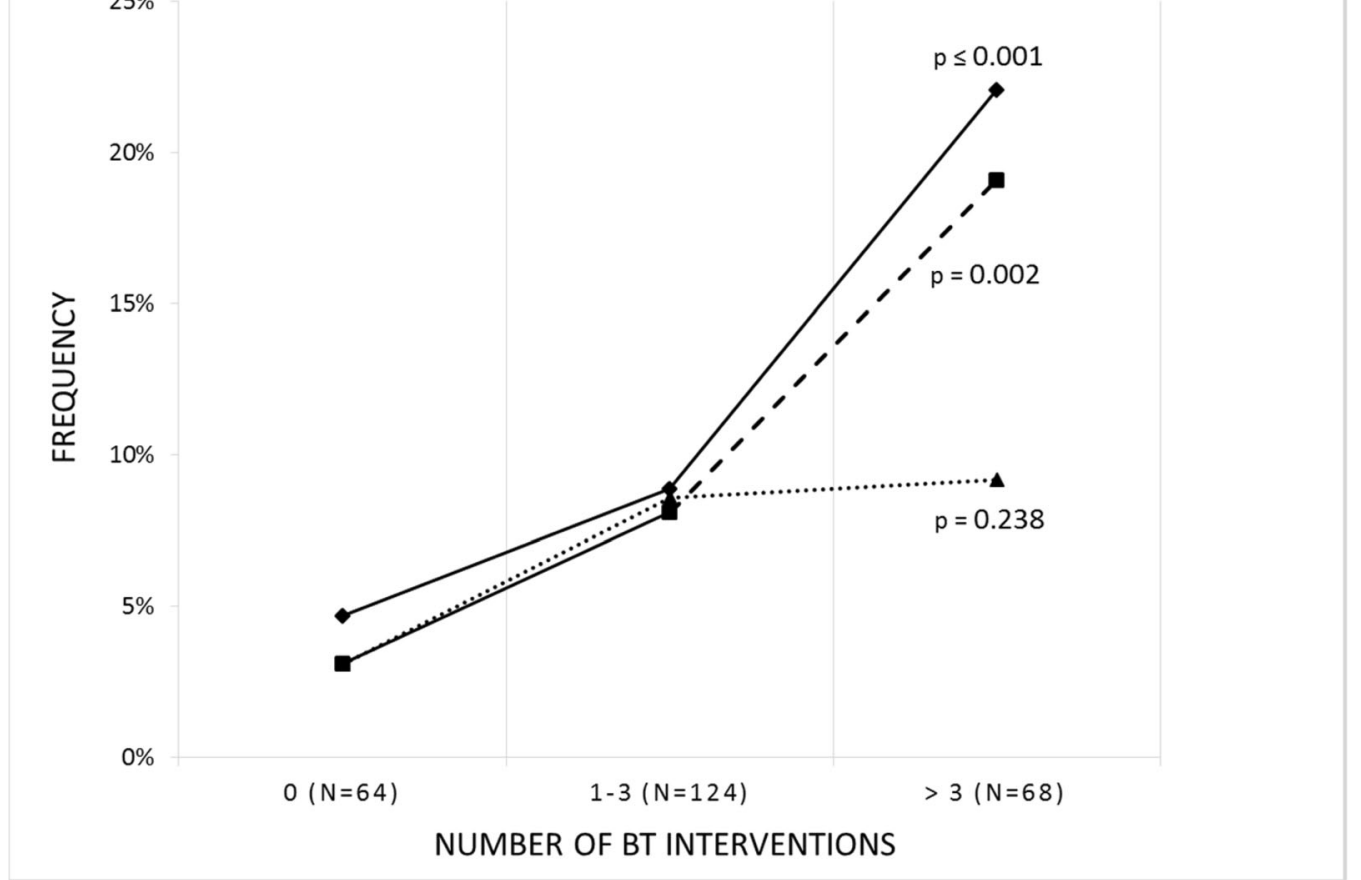

Fig. 2 (See legend on next page.) 
(See figure on previous page.)

Fig. 2 a Linear trends between the number of biliary tract (BT) interventions and the frequency of infections by enterobacterales (diamonds), enterococci (squares), and P.aeruginosa (crosses). $\mathbf{b}$ Linear trends between the number of BT interventions and the frequency of infections by third generation cephalosporine-resistant enterobacterales (3GCRE, diamonds), multi-resistant gram-negative rods (MRGN, squares), and vancomycin-resistant enterococci (VRE, triangles), respectively

pathogens and their expected antimicrobial susceptibility. Accordingly, the current Tokyo guideline recommends third generation cephalosporines, piperacillin/ tazobactam or carbapenems for empirical treatment the choice of which should be guided by local susceptibility data and the severity of the infection. For severe cases and hospital-acquired infections anti-pseudomonal agents and coverage of enterococci are recommended. Because optimal empirical treatment may vary greatly between different institutions, a multi-disciplinary approach is suggested [4].

Although these recommendations provide a useful framework in general, the selection of antibiotic therapy is still associated with various uncertainties. Without guidance by local susceptibility data, it is difficult for the attending physician to make his choice between the three betalactam-options. Carbapenems seem to be the safest choice, because of their bactericidal activity against most gram-negative rods resistant to third generation cephalosporins and acylureidopenicillins. However, increased carbapenem usage promotes carbapenemresistance, which is regarded a major global public health problem. According to recognized principles of antibiotic stewardship, it is therefore widely advocated that carbapenems should be used in a restrictive manner in order to minimize the selection pressure favouring growth of carbapenem-resistant bacteria.

Even if local susceptibility rates are available, they are usually not provided in respect to specific disease entities despite considerable disease-specific variations making the choice of the optimal empirical therapy difficult. Owing to the rising prevalence of resistant gramnegative rods, carbapenems often seem to be the only effective option left. Particularly, empirical therapy guided by microbiological data only may fall short in respect to the goals of modern antibiotic therapy if patient-related response rates are not taken into account. Therefore, the present study aimed to analyse both microbiological as well as patient data in order to optimize empirical therapy of acute cholangitis. To this end, our analysis identified risk factors for 3GCRE and/or MRGN. Although we did find a high overall prevalence of cepholosporine resistant enterobacterales in bile and blood cultures of our patients, our results imply that piperacilline/tazobactam is a highly effective option for patients with communityacquired cholangitis, without pre-existing biliary prosthesis, and not treated on an ICU.
In our cohort, polymicrobial growth occurred in almost $70 \%$ of bile samples with enterobacterales and enterococci being the most common pathogens, each accounting for about one third of bile isolates. Anaerobes were detected in less than $10 \%$ of our patients. These results are consistent with other recent reports [7-10] and revise the classic view that both enterobacterales and anaerobes are the most important pathogens in biliary tract infections [11]. Bacteremia was detected in one third of cases with available blood culture results. Reflecting their differing virulence, and in agreement with a recent large multicenter trial on biliary infections, enterobacterales comprised almost $60 \%$ and enterococci only $20 \%$ of blood culture isolates [2]. In line with previous studies, our multivariate analysis found the presence of a biliary prosthesis an independent predictor for enterococcal isolation from bile $[3,9,12,13]$. The Tokyo guideline recommends empirical treatment covering enterococci in severe community-acquired cholangitis and in health-care associated infections if patients are colonized or enterococci are otherwise of concern. We agree on this but would like to point out that enterococcal coverage should particularly be considered in patients carrying an indwelling biliary drainage. As stated in the guideline, VRE carrier status should be taken into account in the choice of substance [4].

As to the frequency of resistant enterobacterales, we found an $80 \%$ susceptibility of bile isolates to fluorochinolones and an almost $90 \%$ susceptibility of blood isolates to third generation cephalosporines. Thus, on first view, all antibiotic choices recommended by the Tokyo guideline seem to be appropriate for our patients. However, when coverage was analysed in a patient-wise manner, a different picture emerged. Although the antibiotics prescribed largely reflected the Tokyo guideline recommendation, empirical therapy covered bile isolates in only $51 \%$ of patients. In patients with bacteremic infections, coverage was $69 \%$ which is still far less than desired. Probably owing to both the high proportion of polymicrobial growth and the high prevalence of resistant bacteria in bile and blood cultures, the actual antibiotic coverage was unacceptably low.

Epidemiological data from Germany suggest a frequency of 3GCRE of about 15\% in hospitals [14]. In an analysis of cholangitis cases from two other German university hospitals, ESBL producing E.coli were detected at a rate of $31 \%$ [10]. In our cohort, the highest numbers of cholangitis associated with either 3GCRE or MRGN 
Table 5 Empirical antibiotic treatment

\begin{tabular}{|c|c|c|}
\hline & \multicolumn{2}{|c|}{ Frequency } \\
\hline & & (n) \\
\hline \multicolumn{3}{|c|}{ Mono vs. combinational therapy } \\
\hline Mono & $59.4 \%$ & 136 \\
\hline Combinational & $40.6 \%$ & 93 \\
\hline 2 drugs & $33.6 \%$ & 77 \\
\hline$\geq 3$ drugs & $7.0 \%$ & 16 \\
\hline \multicolumn{3}{|c|}{ Prescribed antibiotic substances } \\
\hline Cephalosporine $^{a}$ & $25.8 \%$ & 59 \\
\hline Piperazillin/tazobactam & $23.6 \%$ & 54 \\
\hline Fluorochinolon & $21.4 \%$ & 49 \\
\hline Carbapenem & $18.8 \%$ & 43 \\
\hline Metronidazole & $18.8 \%$ & 43 \\
\hline Ampicillin/sulbactam & $16.2 \%$ & 37 \\
\hline Vancomycin & $9.2 \%$ & 21 \\
\hline Other & $14.8 \%$ & 34 \\
\hline
\end{tabular}

A total of 229 patients were studied

${ }^{a} 2$ nd or 3rd generation

were found amongst patients carrying MDR pathogens. In those known to be colonized, MRGN were detected in $41 \%$ of bile cultures. Individuals with a high burden of comorbidity were also more prone to infections with 3GCRE. Furthermore, the presence of biliary stenosis was associated with an increased risk for MRGN, and we observed a linear trend between the proportion of patients infected with 3GCRE and MRGN and the number of prior biliary tract interventions. Thus, it seems reasonable to cover patients with carrier status, a high burden of comorbitiy or the presence of biliary stenosis and repeated biliary interventions (in our study $>3$ interventions) with a carbapenem for empiric therapy. In addition to these risk factors, we found male sex to be an independent risk factor for isolation of 3GCRE or MRGN. In fact, a previous study on acute cholangitis from two German university centers also reported an association of male sex with infections caused by ESBL E.coli [10]. At the time being, the causal relationship for this association is largely unclear. Therefore, we would presently not suggest to cover all male cholangitis patients with a carbapenem.

Having defined predictors for infection with 3GCRE or MRGN, the question arises, whether patient subgroups can be identified that can still be effectively treated with a carbapenem-sparing therapy even in a tertiary referential center with a high prevalence of resistant pathogens. According to our data, piperacillin/tazobactam would represent a highly effective option for patients presenting with community acquired cholangitis, without indewelling biliary drainage, and not on an ICU. Of note, if only resistance data would have been considered (frequency of 3GCRE in blood culture isolates 11.4\%), the choice would have been a third generation cephalosporine. However, if analysed in a patient-based manner, the coverage by cefotaxime was poor $(<80 \%)$ for unselected patients as well as for all subgroups investigated. The better results for piperacillin/tazobactam in our cohort are explained by the coverage of ampicillin-susceptible enterococci, piperacillin-susceptible P.aeruginosa and anaerobes but not 3GCRE that were tested susceptible to piperacillin/tazobactam in vitro. This is important because piperacillin/tazobactam was inferior to carbapenems in the treatment of blood stream infections caused by E.coli and K.pneumoniae resistant to third generation cephalosporines [15]. Aside from these considerations, our results demonstrate that i) efficacy of antibiotic regimens should be assessed in a patient-based manner, and ii) combined analysis of microbiological and patient data is a valuable tool for deliberate and efficacious use of antibiotics in settings with a high prevalence of resistance.

Since this paper focuses on antibiotic therapy, we have not included a risk analysis for biliary candidiasis. For completeness, a high Charlson Index, bile sampling later

Table 6 Patient-based coverage of blood culture pathogens

\begin{tabular}{|c|c|c|c|c|c|c|c|c|}
\hline \multirow[t]{2}{*}{ Risc factor } & & \multicolumn{3}{|c|}{ CTX } & \multicolumn{2}{|l|}{ TZP } & \multicolumn{2}{|l|}{$\mathrm{CP}$} \\
\hline & & $\mathbf{n}$ & & $p$-value & & $p$-value & & $p$-value \\
\hline \multirow[t]{2}{*}{ Time of bile collection from admission } & $\leq 48 \mathrm{~h}$ & 21 & $71.4 \%$ & 0.062 & $81.0 \%$ & 0.044 & $95.2 \%$ & 0.101 \\
\hline & $>48 \mathrm{~h}$ & 23 & $43.5 \%$ & & $52.2 \%$ & & $78.3 \%$ & \\
\hline \multirow[t]{2}{*}{$\mathrm{ICU}^{+}$} & no & 29 & $69.0 \%$ & 0.024 & $75.9 \%$ & 0.053 & $89.7 \%$ & 0.376 \\
\hline & yes & 15 & $33.3 \%$ & & $46.7 \%$ & & $80.0 \%$ & \\
\hline \multirow[t]{2}{*}{ Indwelling biliary tract drainage } & no & 22 & $68.2 \%$ & 0.128 & $81.8 \%$ & 0.026 & $90.9 \%$ & 0.380 \\
\hline & yes & 22 & $45.5 \%$ & & $50.0 \%$ & & $81.8 \%$ & \\
\hline \multirow[t]{2}{*}{ Any of the above risk factors } & no & 9 & $77.8 \%$ & 0.155 & $100.0 \%$ & 0.016 & $100.0 \%$ & 0.181 \\
\hline & yes & 35 & $51.4 \%$ & & $57.1 \%$ & & $82.9 \%$ & \\
\hline
\end{tabular}

Percentages indicate the proportion of patients for whom all blood culture pathogens would have been covered by the respective antibiotic CTX cefotaxime, TZP piperacillin/tazobactam, CP carbapenem

${ }^{\dagger} \mathrm{ICU}$, treatment on ICU before or during bile sampling 
than $48 \mathrm{~h}$ after admission, and an indwelling drainage were independent risk factors for a fungibilia in our cohort. An indwelling drainage and/or ICU treatment were risk factors for candidaemia .

An important limitation of this retrospective study is the lack of documented information regarding administration of antibiotic therapies prior to microbiological sampling. Owing to its potential effect on microbial spectra and prevelance of resistancies, prior antibiotic exposure should be considered when selecting an empiric regimen. Still, the predictors for a carbapenemsparing therapy identified in our study can be applied to patients without recent antibiotic therapy and to those in which information on antibiotic exposure cannot be reliably obtained from the patient's history.

\section{Conclusion}

In summary, we found a high prevalence of 3GCRE or even MRGN in bile and blood samples obtained from patients with acute cholangitis as would have been expected for a tertiary referential center with associated liver transplant unit. Carrier status, a high burden of comorbitiy, the presence of biliary stenosis, and repeated biliary tract interventions were identified as risk factors for isolation of resistant gram negative bile culture isolates. Only the combined analysis of microbiological and patient data revealed piperacillin/tazobactam as an excellent choice for empiric therapy of patients with community acquired cholangitis, no biliary prosthesis, and no previous or ongoing ICU treatment. The study thus provides an example of how the Tokyo guideline recommendations can be specified for a particular institution on the basis of local microbiology and individual patient characteristics.

\section{Abbreviations \\ 3GCRE: Enterobacterales resistant to third generation cephalosporines; CoNS: Coagulase-negative Staphylococci; ESBL: Extended spectrum beta- lactamase; KRINKO: Commission for Hospital Hygiene and Infection Prevention at the Robert Koch Institute; MDR: Multi-drug resistant; MRGN: Multi-resistant gram-negative rods; VRE: Vancomycin-resistant enterococci}

\section{Acknowledgements}

Not applicable.

\section{Authors' contributions}

TK and HJE developed the concept and design of the study. TA, TK and SGJ collected the microbiological and clinical data. TK and SGJ performed the data analysis. The manuscript was edited by TK, SGJ, TA, BS and HJE. All authors read and approved the final manuscript.

\section{Funding}

The authors received no specific funding for this work.

\section{Ethics approval and consent to participate}

The study was approved by the internal review board (local ethics committee of Charité - Universitätmedizin Berlin; registry number EA2/033/

16). The need for informed consent was waived for this retrospective study.

\section{Consent for publication}

Not applicable.

\section{Competing interests}

The authors declare that they have no competing interests.

Received: 11 September 2019 Accepted: 21 February 2020

Published online: 12 March 2020

\section{References}

1. Lee JG. Diagnosis and management of acute cholangitis. Nat Rev Gastroenterol Hepatol. 2009;6(9):533-41.

2. Gomi H, Takada T, Hwang TL, Akazawa K, Mori R, Endo I, et al. Updated comprehensive epidemiology, microbiology, and outcomes among patients with acute cholangitis. J Hepatobiliary Pancreat Sci. 2017;24(6):310-8.

3. Ortega M, Marco F, Soriano A, Almela M, Martínez JA, López J, et al. Epidemiology and prognostic determinants of bacteraemic biliary tract infection. J Antimicrob Chemother. 2012;67(6):1508-13.

4. Gomi H, Solomkin Joseph S, Schlossberg D, et al. Tokyo guidelines 2018: antimicrobial therapy for acute cholangitis and cholecystitis. J Hepatobiliary Pancreat Sci. 2018;25(1):3-16.

5. Morrissey I, Hackel M, Badal R, Bouchillon S, Hawser S, Biedenbach D. A review of ten years of the Study for Monitoring Antimicrobial Resistance Trends (SMART) from 2002 to 2011. Pharmaceuticals. 2013;6:1335-46.

6. Hygienemaßnahmen bei Infektionen oder Besiedlung mit multiresistenten gramnegativen Stäbchen. Bundesgesundheitsblatt. 2012;55:1311-54.

7. Negm AA, Schott A, Vonberg RP, Weismueller TJ, Schneider AS, Kubicka S, et al. Routine bile collection for microbiological analysis during cholangiography and its impact on the management of cholangitis. Gastrointest Endosc. 2010;72(2):284-91.

8. Goo JC, Hyuk Seong M, Kwang Shim Y, Seung Lee H, Han JH, Seob Shin K, et al. Extended spectrum- $\beta$-lactamase or carbapenemase producing bacteria isolated from patients with acute cholangitis. Clin Endosc. 2012;45(2):15560.

9. Weber A, Schneider J, Wagenpfeil S, Winkle P, Riedel J, Wantia N, et al. Spectrum of pathogens in acute cholangitis in patients with and without biliary endoprosthesis. J Inf Secur. 2013;67(2):111-21.

10. Reuken PA, Torres D, Baier M, Löffler B, Lübbert C, Lippmann N, et al. Risk Factors for Multi-Drug Resistant Pathogens and Failure of Empiric First-Line Therapy in Acute Cholangitis. PLoS ONE. 2017;12(1):e0169900.

11. Brook I. Aerobic and anaerobic microbiology of biliary tract disease. J Clin Microbiol. 1989;27(10):2373-5.

12. Schneider J, Hapfelmeier A, Fremd J, Schenk P, Obermeier A, Burgkart R, et al. Biliary endoprosthesis: A prospective analysis of bacterial colonization and risk factors for sludge formation. PLoS One. 2014;9(10).

13. Lübbert C, Wendt K, Feisthammel J, Moter A, Lippmann N, Busch T, et al. Epidemiology and resistance patterns of bacterial and fungal colonization of biliary plastic stents: A prospective cohort study. PLoS One. 2016;11(5).

14. Bundesamt fur Verbraucherschutz und Lebensmittelsicherheit, Paul-EhrlichGesellschaft fur Chemotherapie e.V. GERMAP 2015 - Bericht uber den Antibiotikaverbrauch und die Verbreitung von Antibiotikaresistenzen in der Human- und Veterinarmedizin in Deutschland. Antiinfectives Intelligence, Rheinbach, 2016.

15. Harris PNA, Tambyah PA, Lye DC, Mo Y, Lee TH, Yilmaz M, et al. Effect of piperacillin-tazobactam vs meropenem on 30-day mortality for patients with e coli or Klebsiella pneumoniae bloodstream infection and ceftriaxone resistance. JAMA. 2018;320(10):984-94.

\section{Publisher's Note}

Springer Nature remains neutral with regard to jurisdictional claims in published maps and institutional affiliations. 